Arch. Math. 83 (2004) 461-466

0003-889X/04/050461-06

DOI 10.1007/s00013-004-1080-5

(C) Birkhäuser Verlag, Basel, 2004

Archiv der Mathematik

\title{
Toral classes and the Gromov-Lawson-Rosenberg Conjecture for elementary abelian 2-groups
}

\author{
By
}

MiCHAEL JOACHIM

\begin{abstract}
Let $\pi$ be a finite elementary abelian 2-group and let $\Omega_{n}(B \pi)$ denote the corresponding spin bordism group. We show that the so-called toral bordism classes in $\Omega_{n}(B \pi)$ for $n \geqq 3$ can be represented by singular manifolds which admit a metric of positive scalar curvature.
\end{abstract}

1. Introduction. In this little note we study an aspect of the so-called Gromov-LawsonRosenberg Conjecture. The Gromov-Lawson-Rosenberg Conjecture for a group $\pi$ states the following

Conje c ture (Gromov-Lawson-Rosenberg Conjecture). Let $M$ be a closed connected spin manifold of dimension $n \geqq 5$ whose fundamental group is isomorphic to $\pi$. Then $M$ admits a metric of positive scalar curvature if and only if the so-called $\alpha$-invariant $\alpha(M) \in K_{n}\left(C_{r}^{*} \pi\right)$ is trivial.

Here $C_{r}^{*} \pi$ denotes the reduced real group $C^{*}$-algebra of $\pi$. In the case where $\pi$ is finite it is isomorphic to the real group algebra $\mathbb{R} \pi$; in the general case it is given by a certain completion of the latter. Since we will not need the explicit definition of the $\alpha$-invariant in this article we omit its definition here and refer the interested reader to $[4,3.4]$ and $[8,4.2]$.

The Gromov-Lawson-Rosenberg Conjecture was motivated by a bordism result which was independently found by Gromov and Lawson [1] and Schoen and Yau [7]. The bordism result has been used to show the Bordism invariance Theorem below. To state it we introduce for a group $\pi$ and an integer $n$ the following subgroup of the corresponding spin bordism group $\Omega_{n}(B \pi)$

$$
\Omega_{n}^{+}(B \pi)=\left\{\begin{array}{l}
\text { bordism classes of } \\
\text { singular } n \text {-dimensional spin manifolds } f: M \rightarrow B \pi, \\
\text { where } M \text { admits a metric of positive scalar curvature }
\end{array}\right\} .
$$

Mathematics Subject Classification (2000): 53C20, 55N22, 55U25. 
Theorem (Bordism invariance, cf.[8, 3.5]). Let $M$ be closed connected spin manifold of dimension $n \geqq 5$ whose fundamental group is isomorphic to a given group $\pi$, and let $c_{M}: M \rightarrow B \pi$ be a map classifying the universal covering of $M$ (i.e. $c_{M}$ is a 2-equivalence). Then $M$ admits a positive scalar curvature metric if and only if

$$
\left[c_{M}: M \rightarrow B \pi\right] \in \Omega_{n}^{+}(B \pi) .
$$

The Bordism Invariance Theorem is related to the Gromov-Lawson-Rosenberg Conjecture as there is a homomorphism $\alpha_{n}: \Omega_{n}(B \pi) \rightarrow K_{n}\left(C_{r}^{*} \pi\right)$ such that $\alpha(M)=$ $\alpha_{n}\left(\left[c_{M}: M \rightarrow B \pi\right]\right)$. By a result of Rosenberg [2, 3.3], [4, 3.4] one knows that $\Omega_{n}^{+}(B \pi) \subset \operatorname{Ker}\left(\alpha_{n}\right)$. So the Gromov-Lawson-Rosenberg Conjecture is equivalent to showing that $\operatorname{Ker}\left(\alpha_{n}\right)=\Omega_{n}^{+}(B \pi)$.

The Gromov-Lawson-Rosenberg Conjecture has been verified for some groups. For example it has been proved to be true for cyclic groups, for groups of finite order with periodic cohomology, also for free and free abelian groups and some more. On the other hand, by now one knows that the conjecture does not hold in general. The first counterexample was given by Schick [6] who showed that the conjecture does not hold for the group $\pi=\mathbb{Z}^{4} \times \mathbb{Z} / 3$. In this case $K_{5}(\mathbb{R} \pi)$ does not contain 3-torsion and Schick showed that a particular 3-torsion element of the form $\left[c_{M}: M \rightarrow B \pi\right] \in \Omega_{5}(B \pi)$ is not contained in $\Omega_{5}^{+}(B \pi)$. The relevant element is a so-called toral class and it has been conjectured that toral classes also might provide counterexamples to the Gromov-Lawson-Rosenberg Conjecture for finite groups. In fact the toral classes are regarded as the essential test case for the Gromov-Lawson-Rosenberg Conjecture to be true for finite groups; here the case of elementary abelian $p$-groups is of particular interest.

An element in $\Omega_{n}(B \pi)$ is called toral if it is contained in the subgroup $\Omega_{n}^{\text {toral }}(B \pi) \subset$ $\Omega_{n}(B \pi)$ generated by the toral generators. Here an element is called a toral generator if it can be represented in the following way. Let $\rho: \mathbb{Z}^{n} \rightarrow \pi$ be a group homomorphism, and let $B \rho: B \mathbb{Z}^{n} \rightarrow B \pi$ denote the corresponding map on the level of classifying spaces. We may assume that $B \mathbb{Z}^{n}$ is given by the $n$-torus $T^{n}$. After choosing a spin structure the map $B \rho: T^{n}=B \mathbb{Z}^{n} \rightarrow B \pi$ defines a singular spin manifold, and elements in $\Omega_{n}(B \pi)$ which can be represented in this way are the toral generators.

Theorem A. Let $\pi$ be an elementary abelian 2-group and let $n \geqq 3$. Then

$$
\Omega_{n}^{\text {toral }}(B \pi) \subset \Omega_{n}^{+}(B \pi) .
$$

In particular the result says that toral classes do not provide counterexamples to the Gromov-Lawson-Rosenberg Conjecture for finite elementary abelian 2-groups. Unfortunately the argument does not generalize to elementary groups of odd order.

2. Proof of Theorem A. First we want to reduce to the case $n=3$. Assume $n \geqq 3$ and let $C_{2}$ denote the elementary abelian 2-group of rank 1 . Then we have a homotopy equivalence $B C_{2}^{n} \cong B C_{2}^{3} \times B C_{2}^{n-3}$.

Proposition. Let $X, Y$ be spaces and let $k, l$ be integers. Taking the product of singular spin manifolds induces a homomorphism

$$
\Omega_{k}^{+}(X) \times \Omega_{l}(Y) \longrightarrow \Omega_{k+l}^{+}(X \times Y) .
$$


Proof. The proposition is well-known. If $f: M \rightarrow X$ and $g: N \rightarrow Y$ are singular spin manifolds their cartesian product is given by $f \times g: M \times N \rightarrow X \times Y$. If $M$ and $N$ are equipped with metrics and the metric of $M$ has positive scalar curvature then one can "shrink" the metric on the factor $M$ so that the scalar curvature at a point $(x, y) \in M \times N$ is dominated by the scalar curvature at $x \in M$.

By the very definition of "toral generator" it follows that a toral generator in $\Omega_{n}\left(B C_{2}^{n}\right)$ is in the image of the cartesian product map

$$
\Omega_{3}^{\text {toral }}\left(B C_{2}^{3}\right) \times \Omega_{n-3}^{\text {toral }}\left(B C_{2}^{n-3}\right) \longrightarrow \Omega_{n}\left(B C_{2}^{n}\right) .
$$

By the previous proposition it hence suffices to show that $\Omega_{3}^{\text {toral }}\left(B C_{2}^{3}\right) \subset \Omega_{3}^{+}\left(B C_{2}^{3}\right)$. To prove the latter we will show

Lemma 1. We have $\Omega_{3}^{+}\left(B C_{2}^{3}\right)=\Omega_{3}\left(B C_{2}^{3}\right)$.

It is well-known that $\Omega_{3}^{+}\left(B C_{2}\right)=\Omega_{3}\left(B C_{2}\right)$. In fact, the whole group $\Omega_{3}\left(B C_{2}\right)$ is cyclic and generated by a singular lens space. The statement of Lemma 1 therefore follows from

Lemma 2. Let $\mathcal{C}$ be the set of cyclic subgroups of $C_{2}^{3}$. Then the induction homomorphism induced by the canonical maps $B C \rightarrow B C_{2}^{3}, C \in \mathcal{C}$

$$
\bigoplus_{C \in \mathcal{C}} \Omega_{3}(B C) \rightarrow \Omega_{3}\left(B C_{2}^{3}\right)
$$

is surjective.

We may study the induction homomorphism of Lemma 2 by an investigation of the Atiyah-Hirzebruch spectral sequence. Recall that the $E^{2}$-term of the spectral sequence converging to $\Omega_{n}(X)$ for a space $X$ is given by $E_{p, q}^{2}=H_{p}\left(X ; \Omega_{q}\right)$, where $\Omega_{q}$ denotes the $q$-th spin bordism group of a point. Let us write $E_{p, q}^{r}(X)$ for the group of bidegree $(p, q)$ in the $E^{r}$-term of the spectral sequence. For $C_{2}$ we have $E_{p, q}^{2}\left(B C_{2}\right)=E_{p, q}^{\infty}\left(B C_{2}\right)$ if $p+q=3$. This follows from the fact that the order of the groups $\Omega_{3}\left(B C_{2}\right)$ and $\oplus_{p+q}=3 H_{p}\left(B C_{2} ; \Omega_{q}\right)$ are the same. Hence to obtain the result of Lemma 2 we simply need to verify that the maps $B C \rightarrow B C_{2}^{3}$ induce surjections $\oplus_{C} H_{p}\left(B C ; \Omega_{q}\right) \rightarrow H_{p}\left(B C_{2}^{3} ; \Omega_{q}\right)$ for all $(p, q)$ with $p+q=3$ which we formulate as

Lemma 3. The canonical maps $B C \rightarrow B C_{2}^{3}$ for $C \in \mathcal{C}$ induce surjections

(a) $\bigoplus_{C} H_{3}(B C ; \mathbb{Z}) \longrightarrow H_{3}\left(B C_{2}^{3} ; \mathbb{Z}\right)$,

(b) $\bigoplus_{C} H_{2}(B C ; \mathbb{Z} / 2) \longrightarrow H_{2}\left(B C_{2}^{3} ; \mathbb{Z} / 2\right)$,

(c) $\bigoplus_{C} H_{1}(B C ; \mathbb{Z} / 2) \longrightarrow H_{1}\left(B C_{2}^{3} ; \mathbb{Z} / 2\right)$.

We shall consider the first statement first. Let $J$ be a non-empty subset of the set $\{1,2,3\}$. For any such subset $J$ define $\rho_{J}: C_{2} \rightarrow C_{2}^{3}$ so that $\operatorname{pr}_{i} \circ \rho_{J}=\operatorname{id}_{C_{2}}$ if $i \in J$ and $\operatorname{pr}_{i} \circ \rho_{J}$ is trivial if $i \notin J$, where $\operatorname{pr}_{i}: C_{2}^{3} \rightarrow C_{2}$ denotes the projection onto the $i$-th factor. For each 
$J$ the image of $\rho_{J}$ is a cyclic subgroup $C_{J}$ of order 2. By abuse of notation let $\rho_{J}: C_{J} \rightarrow C_{2}^{3}$ also denote the inclusion of the subgroup $C_{J}$ into $C_{2}^{3}$. We want to prove that the induced maps $B \rho_{J}: B C_{J} \rightarrow B C_{2}^{3}$ induce an isomorphism

$$
\bigoplus_{J} H_{3}\left(B C_{J} ; \mathbb{Z}\right) \longrightarrow H_{3}\left(B C_{2}^{3} ; \mathbb{Z}\right) .
$$

To do this consider for each $J$ the composition

$$
p_{J}: B C_{2}^{3} \longrightarrow \prod_{j \in J} B C_{\{j\}} \rightarrow \bigwedge_{j \in J} B C_{\{j\}}=: B(J) .
$$

Lemma 4. For two non-empty subsets $J, J^{\prime} \subset\{1,2,3\}$ the composition

$$
H_{3}\left(p_{J^{\prime}} \circ B \rho_{J} ; \mathbb{Z}\right): H_{3}\left(B C_{J} ; \mathbb{Z}\right) \rightarrow H_{3}\left(B C_{2}^{3} ; \mathbb{Z}\right) \rightarrow H_{3}\left(B\left(J^{\prime}\right) ; \mathbb{Z}\right)
$$

is an isomorphism if $J^{\prime} \subset J$, and it is trivial if $J^{\prime} \not \subset J$.

Pro of. We may use the Künneth theorem and the stable splittings

$$
\begin{aligned}
& B C_{2}^{2} \cong_{s} B C_{2} \vee B C_{2} \vee B C_{2} \wedge B C_{2} ; \\
& B C_{2}^{3} \cong_{s} \bigvee_{J^{\prime}} B\left(J^{\prime}\right)
\end{aligned}
$$

to conclude that $H_{3}\left(B\left(J^{\prime}\right) ; \mathbb{Z}\right) \cong \mathbb{Z} / 2$. We now consider the diagram

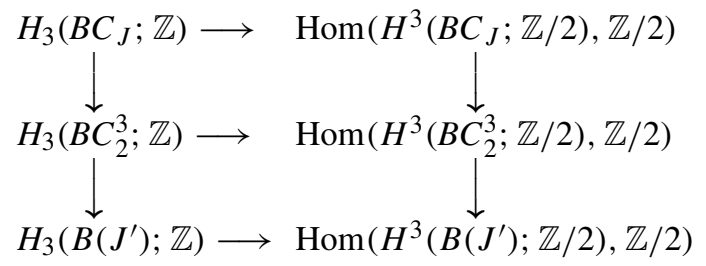

where the vertical arrows are induced by $p_{J^{\prime}}$ and $B \rho_{J}$ respectively, and where the horizontal arrow at each case is given by the composition of the reduction map $H_{3}(\quad ; \mathbb{Z}) \rightarrow$ $H_{3}(; \mathbb{Z} / 2)$ with the duality isomorphism $H_{3}(; \mathbb{Z} / 2) \rightarrow \operatorname{Hom}\left(H^{3}(; \mathbb{Z} / 2), \mathbb{Z} / 2\right)$. Note that the horizontal arrows are all injective. Since $H_{3}\left(B C_{J} ; \mathbb{Z}\right)$ and $H_{3}\left(B\left(J^{\prime}\right) ; \mathbb{Z}\right)$ are both isomorphic to $\mathbb{Z} / 2$, the composition of vertical arrows on the left hand side (which is the homomorphism we are after) is either trivial or an isomorphism. Note also, since the top horizontal arrow $H_{3}\left(B C_{J} ; \mathbb{Z} / 2\right) \rightarrow \operatorname{Hom}\left(H^{3}\left(B C_{J} ; \mathbb{Z} / 2\right), \mathbb{Z} / 2\right)$ is an isomorphism and since the bottom horizontal arrow is injective, we equally well may check whether the composition of the vertical arrows on the right hand side is trivial or not. Since $\operatorname{Hom}\left(H^{3}\left(B C_{J} ; \mathbb{Z} / 2\right), \mathbb{Z} / 2\right) \cong \mathbb{Z} / 2$, this is equivalent to checking whether this composition is trivial or not; or dually we can check whether the composition $H^{3}\left(B \rho_{J} ; \mathbb{Z} / 2\right) \circ$ $H^{3}\left(p_{J^{\prime}} ; \mathbb{Z} / 2\right)$ is trivial or not. 
As is well-known we have isomorphisms $H^{*}\left(B C_{J} ; \mathbb{Z} / 2\right) \cong \mathbb{Z} / 2\left[x_{J}\right]$ and $H^{*}\left(B C_{2}^{3} ; \mathbb{Z} / 2\right) \cong \mathbb{Z} / 2\left[x_{1}, x_{2}, x_{3}\right]$; here $x_{J} \in H^{1}\left(B C_{J} ; \mathbb{Z} / 2\right)$ denotes the unique generator, and the $x_{i}$ are given by $x_{i}=p r_{i}^{*} x$ where the element $x$ is the unique generator in $H^{1}\left(B C_{2} ; \mathbb{Z} / 2\right)$. The homomorphism $H^{*}\left(B \rho_{J} ; \mathbb{Z} / 2\right): H^{*}\left(B C_{2}^{3} ; \mathbb{Z} / 2\right) \rightarrow H^{*}\left(B C_{J} ; \mathbb{Z} / 2\right)$ is given by $x_{i} \mapsto x_{J}$ if $i \in J$ and $x_{i} \mapsto 0$ if $i \notin J$, while the homomorphism $H^{*}\left(B p_{J^{\prime}} ; \mathbb{Z} / 2\right)$ : $H^{*}\left(B\left(J^{\prime}\right) ; \mathbb{Z} / 2\right) \rightarrow H^{*}\left(B C_{2}^{3} ; \mathbb{Z} / 2\right)$ is given by the inclusion of the subgroup generated by all monomials of the form $x_{i_{1}}^{\alpha_{1}} \cdots x_{i_{r}}^{\alpha_{r}}$ with $\alpha_{j} \geqq 1$ for all $1 \leqq j \leqq r$ and $i_{1}<\ldots<i_{r}$ and $\left\{i_{1}, \ldots, i_{r}\right\}=J^{\prime}$. From this description we see that the composition $H^{3}\left(B \rho_{J} ; \mathbb{Z} / 2\right) \circ H^{3}\left(p_{J^{\prime}} ; \mathbb{Z} / 2\right): H^{3}\left(B\left(J^{\prime}\right) ; \mathbb{Z} / 2\right) \rightarrow H^{3}\left(B C_{J} ; \mathbb{Z} / 2\right)$ is non-trivial if and only if $J^{\prime} \subset J$.

We now consider the composition

$$
\bigoplus_{J} H_{3}\left(B C_{J} ; \mathbb{Z}\right) \stackrel{l}{\longrightarrow} H_{3}\left(B C_{2}^{3} ; \mathbb{Z}\right) \stackrel{r}{\longrightarrow} \prod_{J} H_{3}\left(B\left(J^{\prime}\right) ; \mathbb{Z}\right),
$$

where $l$ is given by the maps $H_{3}\left(B \rho_{J} ; \mathbb{Z}\right)$ and where $r$ is given by the maps $H_{3}\left(p_{J^{\prime}} ; \mathbb{Z}\right)$. Domain and target of this composition are both indexed over the set of $J$ 's, while the individual groups $H_{3}\left(B C_{J} ; \mathbb{Z}\right)$ and $H_{3}\left(B\left(J^{\prime}\right) ; \mathbb{Z}\right)$ that occur as summands and factors respectively are isomorphic to $\mathbb{Z} / 2$. Using an ordering of the set of $J$ 's therefore allows to present the composition by a matrix. For the ordering $\{1\},\{2\},\{3\},\{1,2\},\{1,3\},\{2,3\}$, $\{1,2,3\}$ this matrix is given by

$$
\left(\begin{array}{lllllll}
1 & 0 & 0 & 1 & 1 & 0 & 1 \\
0 & 1 & 0 & 1 & 0 & 1 & 1 \\
0 & 0 & 1 & 0 & 1 & 1 & 1 \\
0 & 0 & 0 & 1 & 0 & 0 & 1 \\
0 & 0 & 0 & 0 & 1 & 0 & 1 \\
0 & 0 & 0 & 0 & 0 & 1 & 1 \\
0 & 0 & 0 & 0 & 0 & 0 & 1
\end{array}\right)
$$

as we can easily calculate from Lemma 4 . The matrix has determinant equal to 1 so the composition $r \circ l$ above is an isomorphism. Hence $l$ is an injection. Now by simply counting the elements of the domain and target of $l$ (using the Künneth theorem) we see that $l$ is an (injective) map between sets of the same finite cardinality. Hence $l$ is in fact an isomorphism. Since the left hand side of the composition is a direct summand of the left hand side of the homomorphism of Lemma 3 (a) this shows that the latter is surjective.

To prove surjectivity for the homomorphisms in Lemma 3 (b) and (c) one can argue in a similar fashion using subsets $J$ which contain at most 2 resp. 1 elements. We shall omit the details here.

This completes the proof of Lemma 3 and thus the proof of Theorem A. 


\section{References}

[1] M. GROMOV and H. B. LAwsOn, The classification of simply-connected manifolds of positive scalar curvature. Ann. of Math. 111, 423-434 (1980).

[2] J. RosEnBERG, $C^{*}$-algebras, positive scalar curvature, and the Novikov conjecture. Publ. Math. IHES 58, 197-212 (1983).

[3] J. RosEnBERG, $C^{*}$-algebras, positive scalar curvature, and the Novikov conjecture II. In: Geometric methods in operator algebra. H. Araki and E. G. Effros, eds., Pitman Res. Notes Math. 123, 341-374 (1986).

[4] J. RosenberG, $C^{*}$-algebras, positive scalar curvature, and the Novikov conjecture III. Topology 25, 319-336 (1986).

[5] J. Rosenberg and S. Stolz, Manifolds of positive scalar curvature. In: Algebraic Topology and its Applications. Math. Sci. Res. Inst. Publ. 27, 241-267 (1994).

[6] T. SCHICK, A counterexample to the (unstable) Gromov-Lawson-Rosenberg conjecture. Topology 37 , 1165-1168 (1998).

[7] R. SCHOEN and S. T. YAU, On the structure of manifolds with positive scalar curvature. Manuscr. Math. 28, 159-183 (1979).

[8] S. STOLZ, Positive scalar curvature metrics-existence and classification. In: Proc. Intern. Congress Math., Zürich, Switzerland 1994. Volume I, 626-636 (1995).

Received: 9 May 2003; revised manuscript accepted: 27 April 2004

Michael Joachim

Mathematisches Institut

Westfälische Wilhelms-Universität Münster

D-48149 Münster

Germany

joachim@math.uni-muenster.de 